Documento No. 25

Un Modelo Financiero de Desequilibrio a Corto Plazo para la Economía Mexicana

por

Alain Ize

Julio, 1980

Este documento forma parte de un proyecto conjunto de El Colegio de México - Banco de México. El autor agradece la muy valiosa asistencia de Angel Calderón en la elaboración del modelo desarrollado en este trabajo.

Las ideas contenidas en el presente son responsabilidad exclusiva del autor y no reflejan la posición del Banco de México, S.A. 


\section{UN MODELO FINANCIERO DE DESEQUILIBRIO \\ A CORTO PLAZO PARA LA ECONOMIA MEXICANA}

por

Alain Ize

\section{Introducción.}

La intermediación financiera en México se caracteriza, a nivel macroeconómico, por dos rasgos particularmente importantes: por una parte, existe una marcada preponderancia del sistema bancario y una insignificancia relativa de la bolsa de valores ${ }^{1}$; por la otra, prevalecen situaciones de desequilibrio en los mercados financieros que gravitan alrededor del sistema bancario. En el mercado de bonos y valores bancarios, el gobierno impone topes sobre todas las tasas pasivas de interés y éstas generalmente se encuentran en estos topes, lo que sugiere que existe, en general, desequilibrio por exceso de oferta. Parece también presentarse en el mercado de fondos prestables un estado casi constante de desequilibrio por exceso de demanda o por exceso de oferta, ya que las tasas activas de interés se ajustan muy lenta y parcialmente ${ }^{2}$.

Por último en el mercado de bonos gubernamentales, la adquisición de bonos está en gran parte condicionada por requerimientos de encaje; han empezado a ser emitidos algunos bonos (certificados de tesorería) que pueden ser libremente intercambiados en mercados abiertos, pero su participación es aún modesta y seguirá siéndolo por algún tiempo ${ }^{3}$

La conjunción de los dos rasgos mencionados anteriormente - la preponderancia de la intermediación bancaria y la existencia casi permanente de estados de desequilibrio en los mercados financieros - concede al sistema financiero mexicano una singular caracterización que es necesario expresar adecuadamente en cualquier modelo de política monetaria. Un primer intento en este sentido se encuentra en el artículo de Bazdresch "La Política Monetaria Mexicana: una primera aproximación"

\footnotetext{
${ }^{1}$ Recientemente se ha observado un crecimiento sostenido de la bolsa de valores que podría permitir alcanzar un mayor equilibrio entre ambos canales de financiamiento.

2 Todos los estudios econométricos que han usado tasas activas de interés como regresores de ofertas y demandas de fondos prestables han mostrado una insignificancia persistente de estas tasas (este punto es discutido con más detalle en Ize, 1978).

${ }^{3}$ Sobre la importancia e impacto de los certificados de tesorería, véase Solís (1978).
} 
(1973). El objetivo de este trabajo es el de desarrollar un modelo más completo que represente consistentemente las características de desequilibrio de la economía mexicana, y que constituya una "segunda aproximación" al problema de la política monetaria en México.

En primer término se hará repaso de algunos conceptos claves que han surgido en los últimos quince años en la literatura post-Keynesiana sobre modelos de desequilibrio ${ }^{4}$ y se presentará el modelo que será analizado después. Finalmente se plantearán algunas posibles extensiones del modelo y se examinarán ciertos problemas y requisitos de estimación econométrica.

\section{El Modelo}

\section{II.1 Demandas efectivas y "efectos de derrame"}

El punto de partida para la derivación de funciones de oferta y de demanda es la maximización de alguna función objetivo (función de utilidad en el caso del consumidor, de ganancias en el caso de la empresa) bajo una restricción de equilibrio presupuestal. Si la única restricción existente es la presupuestal y si el agente puede intercambiar tanto como desee de cualquier bien a los precios a que se enfrenta, sus ofertas y demandas son "nocionales" en la terminología de Clower. Para que todos los agentes puedan realizar sus transacciones deseadas es necesario que ofertas y demandas globales sean iguales en todos los mercados, o sea, que exista una situación de equilibrio general.

Al existir desequilibrio en un mercado, algunos agentes no satisfacen sus deseos de compra o de venta. Cuando un agente se enfrenta, además de la restricción presupuestal, a una restricción de cantidades intercambiables, al maximizar su función objetivo, ajusta sus demandas y ofertas nocionales de manera que las cantidades que realmente intercambia en los mercados corresponden a demandas y ofertas efectivas y no nocionales. La restricción percibida en un mercado A tiene un efecto en cualquier otro mercado b que se puede llamar "efecto de derrame" del mercado A al mercado B.

El efecto de derrame refleja la discrepancia entre las ventas y compras planeadas y las realizadas.

\footnotetext{
${ }^{4}$ Algunas de las contribuciones más importantes es este momento son las de Clower (1965), Leijonhufvud (1968) Barro y Grossman (1971) y Malinvaud (1977).
} 
Si $\mathrm{Y}^{d}$ y $\mathrm{X}^{d}$ son las demandas nocionales en los mercados $\mathrm{Y}$ y $\mathrm{X} ; \mathrm{Y}^{*}$ y $\mathrm{X}^{*}$ las demandas efectivas y $\bar{X}$ es una restricción impuesta en el mercado $\mathrm{X}$, una forma conveniente de formular el efecto de derrame es la siguiente:

$$
\begin{aligned}
& \mathrm{Y}^{*}=\mathrm{Y}^{d}+\mathrm{h}\left(\mathrm{X}^{d}-\mathrm{X}^{*}\right) \\
& \mathrm{X}^{*}=\min \left\{X^{d}, \bar{X}\right\}
\end{aligned}
$$

h es un "coeficiente de derrame" del mercado X al mercado $Y$, que puede ser una función del conjunto de precios existente en la economía y de la restricción $\bar{X}$. Para mayor simplicidad se considera aquí que este coeficiente es constante, lo que equivale a suponer que el efecto de derrame es aproximadamente lineal.

Es importante destacar que tanto las demandas nocionales como las efectivas deben satisfacer las restricciones presupuestales de todos los agentes y cumplir, por lo tanto, la ley de Walras. Esto implica, a la vez, que los términos de derrame satisfacen también por sí solos la ley de Walras.

\section{II.2 Características Generales del Modelo}

Se presenta un modelo de corto plazo, entendiéndose por esto, un lapso menor a un año. En México las variables fiscales (gasto público y nivel de imposición fiscal) son difícilmente modificables dentro del año presupuestal por lo que, se consideran como dadas y se dejan fuera del modelo. Por otra parte, se considera un equilibrio de fin de periodo para todos los acervos; si $\mathrm{A}_{1}$ es el valor de un acervo al final del periodo, $\mathrm{A}_{0}$ su valor al inicio y $\mathrm{A}$ es el cambio en el periodo:

$$
\mathrm{A}=\mathrm{A}_{1}-\mathrm{A}_{0}
$$

como $\mathrm{A}_{0}$ está dado, se puede dejar fuera del modelo, identificándose entonces el flujo A con el acervo $\mathrm{A}_{1}$ 
Las variables bajo control gubernamental son, por una parte, el requerimiento de encaje e, y, por otra, la tasa pasiva ${ }^{r} \mathrm{p}^{5}$. Se considera que en el corto plazo la tasa activa sigue simplemente los movimientos de la tasa pasiva, ya que los bancos mantienen cierto margen de ganancia sobre sus operaciones. La tasa de interés sobre bonos gubernamentales no se incluye en el modelo; se considera que no constituye en México un instrumento activo e independiente de control monetario. La tasa de cambio se supone fija en el corto plazo. No se introduce en el modelo ni la inflación interna ni una variable de riesgo cambiario. Ambas variables podrían, sin embargo, ser incluidas sin dificultad. Finalmente, para mayor simplicidad se supone un solo sector productor de bienes.

Los acervos en poder del público, son dinero en efectivo, $(\mathrm{H})$, cuentas bancarias, $(\mathrm{M}) 6$, préstamos otorgados por los bancos nacionales, (L), y préstamos otorgados por bancos extranjeros, (L'). El acervo de valores extranjeros en manos de mexicanos puede ser descontado del acervo de préstamos (L'). Los acervos que maneja el sistema bancario son las mismas cuentas (M), los préstamos (L), bonos gubernamentales, (B), y cuentas y depósitos de extranjeros en bancos mexicanos (D). Se hace la hipótesis que los bancos no mantienen dinero en efectivo y que todas las reservas legales son intercambiadas por bonos gubernamentales. Finalmente, el gobierno emite dinero en efectivo, $(\mathrm{H}), \mathrm{y}$ bonos, (B); mantiene además reservas en divisas $\left(\mathrm{M}_{R}\right)$.

\section{II.3 Ecuaciones de Mercados}

El primer conjunto de ecuaciones del modelo corresponde a las condiciones de equilibrio (o de desequilibrio) en los mercados de bienes y de acervos monetarios y financieros.

$$
\begin{aligned}
& \mathrm{Y}+\mathrm{Z}(\mathrm{Y})=\mathrm{A}^{d}\left(\mathrm{Y}, \mathrm{r}_{p}\right)-\mathrm{h}_{A}\left(\mathrm{~L}^{d}\left(\mathrm{Y}, \mathrm{r}_{p}\right)+\mathrm{L}^{d}\left(\mathrm{Y}, \mathrm{r}_{p}\right)-\mathrm{L}-\mathrm{L}^{\prime}\right) \\
& \quad \mathrm{A}^{d}{ }_{1} \geq 0, \mathrm{~A}^{d}{ }_{2} \leq 0, \mathrm{~L}^{d}{ }_{1} \geq 0, \mathrm{~L}^{d}{ }_{2} \leq 0, \mathrm{~L}^{d_{1}}{ }_{1} \geq 0, \mathrm{~L}^{{ }_{d}}{ }_{2} \geq 0, \mathrm{Z}_{1} \geq 0, \mathrm{~h}^{A} \geq 0 \\
& \mathrm{~L}=\min \left\{L^{d}\left(\mathrm{Y}, \mathrm{r}_{p}\right),(1-\mathrm{e}) \propto(\mathrm{M}+D)\right\} \\
& 0 \leq \propto \leq 1
\end{aligned}
$$

\footnotetext{
${ }^{5}$ Se hace la hipótesis que el mercado de valores bancarios está siempre en desequilibrio por exceso de oferta: mover los topes impuestos sobre las tasas pasivas equivale entonces a mover directamente estas tasas.

${ }^{6}$ Estas pueden ser cuentas de cheques, cuentas de ahorro e inclusive valores o bonos bancarios.
} 


$$
\begin{aligned}
& \mathrm{M}=\mathrm{M}^{d}\left(\mathrm{Y}, \mathrm{r}_{p}\right)+\mathrm{h}_{M}\left(\mathrm{~L}^{d}\left(\mathrm{Y}, \mathrm{r}_{p}\right)+\mathrm{L}^{d}\left(\mathrm{Y}, \mathrm{r}_{p}\right)-\mathrm{L}^{\prime} \mathrm{L}^{\prime}\right) \\
& \mathrm{M}^{d}{ }_{1} \geq 0, \mathrm{M}^{d}{ }_{2} \geq 0, \mathrm{~h}_{M}{ }^{2} 0 \\
& \mathrm{~L}^{\prime}=\mathrm{L}^{\prime}{ }^{d}\left(\mathrm{Y}, \mathrm{r}_{p}\right)+\mathrm{h}_{L^{\prime}}\left(\mathrm{L}^{d}\left(\mathrm{Y}, \mathrm{r}_{p}\right)-\mathrm{L}\right) \\
& 0 \leq \mathrm{h}_{L^{\prime}} \leq 1 \\
& \mathrm{D}=\mathrm{D}^{d}\left(\mathrm{r}_{p}\right) \\
& \mathrm{D}^{d}{ }_{1} \leq 0 \\
& \mathrm{Z}(\mathrm{Y})+\mathrm{M}_{R}=\mathrm{D}+\mathrm{L}^{\prime}
\end{aligned}
$$

(3) es la condición de equilibrio en el mercado de bienes: la oferta de bienes, suma de la oferta interna y de la externa $\mathrm{Z}(\mathrm{Y})$, es igual a la demanda efectiva de bienes, que se obtiene sumando la demanda nacional, $\mathrm{A}^{d}$, y un término de derrame que corresponde al desequilibrio que pueda existir en los mercados de crédito $^{7}$. Se hace la hipótesis que el crédito obtenido por el público puede ser usado en la misma forma, independientemente de que sea crédito interno y externo.

Los signos esperados de las derivadas están dados debajo de la ecuación. Un aumento en la tasa pasiva tiende a elevar la tasa activa, y provoca por lo tanto una substitución entre crédito interno y externo.

(4) es la ecuación de desequilibrio en el mercado interno de fondos prestables. Los fondos disponibles para préstamos corresponden a la parte no sujeta a encaje de los pasivos bancarios (1-e) $(\mathrm{M}+\mathrm{D})$. Los bancos pueden, sin embargo, desear adquirir una mayor cantidad de bonos que los

\footnotetext{
${ }^{7}$ Se está suponiendo que el mercado de bienes está siempre en equilibrio y que no hay efecto de derrame que provenga de este mercado. Un modelo más completo eliminaría esta hipótesis (Korliras (1975) presenta un modelo de este último tipo).
} 
requeridos por el encaje, conservando así más liquidez, como medida de precaución. El coeficiente $\propto$ puede entonces ser menor que uno.

(5) es la ecuación de equilibrio en el mercado de cuentas bancarias. Un mayor racionamiento de crédito puede hacer aumentar la demanda de cuentas bancarias si por motivos de precaución a la vez que de inversión una mayor incertidumbre en la obtención de crédito obliga al público a elevar sus reservas líquidas. Cuando existen fuertes discontinuidades en el proceso de inversión y no se pueden conseguir créditos, el público tiene que acumular fondos hasta que tenga la cantidad suficiente para invertir $^{8}$. Pero es también posible que en el corto plazo, frente a una mayor restricción crediticia, los agentes tengan que reducir sus cuentas bancarias para poder hacer frente a sus obligaciones de pagos. $\mathrm{h}_{M}$ puede, por lo tanto, ser positivo o negativo.

(6) es la condición de equilibrio en el mercado externo de fondos prestables. El efecto de derrame es positivo y proporcional al desequilibrio existente en el mercado interno de fondos. Si ambos tipos de crédito fueran perfectamente substituibles en la preferencia del público, $\mathrm{h}_{L}$ ' sería igual a uno. Sin embargo, los créditos externos están asociados con un riesgo cambiario. Por otra parte, solo ciertas empresas (particularmente las transnacionales) tienen el adecuado acceso a este tipo de crédito. Existen, entonces, restricciones por el lado de la oferta. Para conservar la simplicidad del modelo no se divide al público en dos conjuntos (los que pueden conseguir créditos externos y los que no), sino que se integra la restricción de oferta al lado de la demanda, a través del coeficiente de derrame $\mathrm{h}_{L}$ ' que toma valores menores que uno.

Finalmente, (7) es la condición de equilibrio en el mercado de valores mexicanos demandados por extranjeros; y (8) es la condición de equilibrio en el mercado de divisas.

\section{II.4 Restricciones presupuestales y la ley de Walras}

El segundo conjunto de ecuaciones corresponde a entidades contables que expresan las restricciones presupuestales a las que están sujetos los agentes.

$$
\mathrm{Y}+\mathrm{L}+\mathrm{L}^{\prime}=\mathrm{A}+\mathrm{H}+\mathrm{M}
$$

\footnotetext{
${ }^{8}$ Este es el efecto descrito por Mc Kinnon (1975).
} 


$$
\begin{aligned}
& \mathrm{Y}+\mathrm{L}^{d}+\mathrm{L}^{\prime} d=\mathrm{A}^{d}+\mathrm{H}^{d}+\mathrm{M}^{d} \\
& \mathrm{M}+\mathrm{D}=\mathrm{B}+\mathrm{L} \\
& \mathrm{B}+\mathrm{H}=\mathrm{M}^{R}
\end{aligned}
$$

(9) y (10), corresponden a las restricciones presupuestales del público, expresadas en términos efectivos o nocionales: el flujo de ingreso y de préstamos es gastado o almacenado en forma de dinero circulante o de cuentas bancarias; (11) es la condición de equilibrio entre activos y pasivos del sistema bancario; (12) es la condición de equilibrio presupuestal del gobierno: cambios en las reservas de divisas deben ser cubiertos por cambios equivalentes en la emisión de dinero o de bonos.

Es fácil verificar, usando las ecuaciones (3), (8), (11) y (12) que la ecuación (9) no es independiente. El sistema satisface la ley de Walras en términos efectivos. La ecuación (9) implica a la vez, que la demanda efectiva de dinero circulante, $\mathrm{H}$, está determinada en forma residual a partir de la restricción presupuestal. El equilibrio en el mercado de dinero se deriva, por lo tanto, de las condiciones de equilibrio en los demás mercados; por esta razón no se incluye la ecuación correspondiente dentro del conjunto de ecuaciones de mercado especificadas anteriormente. De igual manera, la demanda "libre" de bonos por parte de los bancos es obtenida como un residuo a partir de la ecuación (11), y se excluye del modelo la condición de equilibrio en este mercado, puesto que debe automáticamente ser satisfecha.

Restando la ecuación (9) de la (10) y usando las ecuaciones (5) y (6), se obtiene la siguiente condición: ${ }^{9}$

$$
\mathrm{h}_{A}=1+\mathrm{h}_{M}
$$

Esta restricción sobre el valor de los términos de derrame es necesaria para que el sistema satisfaga la ley de Walras en términos nocionales. Al cumplirse la condición (13), (10) queda entonces automáticamente satisfecha.

\footnotetext{
${ }^{9}$ Se hace la hipótesis que el término de derrame en la demanda de dinero circulante es muy pequeña y puede despreciarse.
} 
El sistema queda compuesto por 8 ecuaciones independientes, 2 ecuaciones dependientes y un conjunto de restricciones sobre el valor de los términos de derrame.

\section{Análisis}

\section{III.1 El Sistema Reducido}

Este sistema de 8 ecuaciones independientes requiere de 8 variables endógenas. Estas son Y, L, L', M, H, D, B y $\mathrm{M}_{R}$. En particular, se destaca que tanto la emisión de dinero como la de bonos por parte del gobierno son endógenas. El gobierno dispone de dos instrumentos: el encaje y la tasa pasiva, con los cuales trata de alcanzar dos objetivos independientes, por ejemplo, cierto nivel de ingreso y cierto nivel de reservas en divisas.

Para analizar este sistema es conveniente ponerlo en forma reducida del tipo IS - LM. La IS es la ecuación de equi- en el mercado de bienes. Usando (6) y la condición (13), la ecuación (3) se puede escribir:

$$
\mathrm{Y}+\mathrm{Z}(\mathrm{y})=\mathrm{A}^{d}\left(\mathrm{Y}, \mathrm{r}_{p}\right)-\left(1+\mathrm{h}_{M}\right)\left(1-\mathrm{h}_{L^{\prime}}\right)\left(\mathrm{L}^{d}\left(\mathrm{Y}, \mathrm{r}_{p}\right)-\mathrm{L}\right)
$$

La LM se puede obtener a partir de la ecuación de equilibrio ene le mercado de cuentas bancarias y de la de desequilibrio en el mercado de fondos prestables. A partir de esta última ecuación se deduce que:

$$
\mathrm{M}=\mathrm{L} / \propto(1-\mathrm{e})-\mathrm{D}
$$

$$
\text { Si } \mathrm{L}<\mathrm{L}^{d}\left(\mathrm{Y}, \mathrm{r}_{p}\right)
$$

Cuando se satisface la condición (16) existe desequilibrio por exceso de demanda en el mercado de fondos prestables y la LM es entonces:

$$
\mathrm{L} /(1-\mathrm{e}) \propto=\mathrm{D}\left(\mathrm{r}_{p}\right)+\mathrm{M}^{d}\left(\mathrm{Y}, \mathrm{r}_{p}\right)+\mathrm{h}_{M}\left(1-\mathrm{h}_{L^{\prime}}\right)\left(\mathrm{L}^{d}\left(\mathrm{Y}, \mathrm{r}_{p}\right)-\mathrm{L}\right)
$$


Esta ecuación corresponde a una condición de equilibrio financiero entre los fondos que entran y los que salen del sector bancario. Cuando la condición (16) se verifica, las ecuaciones (14) y (17) definen entonces el estado interno de la economía, o sea el nivel de ingreso, $\mathrm{Y}^{*}$, y la cantidad de fondos prestados, $\mathrm{L}^{*}$. Cuando (16) no se verifica, el estado de la economía se define a partir de la ecuación (14) y de la condición $\mathrm{L}=\mathrm{L}^{d}$. La ecuación (8) define, por otra parte el estado externo de la economía, o sea el nivel de reservas en divisas. Las ecuaciones (8), (14) y (17), junto con la condición (16), constituyen un sistema reducido de tres variables endógenas: $\mathrm{Y}, \mathrm{L}$ y $\mathrm{M}_{R}$.

\section{III.2 Un análisis gráfico.}

Este sistema reducido puede ser analizado gráficamente en el plano (Y, L). La condición (16) define un área del plano en el cual la economía debe permanecer. Dado que la demanda nocional de préstamos aumenta con el nivel de ingreso, la pendiente de esta línea de frontera es positiva. Diferenciando la ecuación (14), se puede derivar la pendiente de la IS:

$$
\frac{d L}{d Y} / \overline{\bar{I} S} \frac{1+Z_{1+\left(1+h_{M}\right)\left(1-h_{L}\right) L_{1}^{d}-A_{1}^{d}}}{\left(1+h_{M}\right)\left(1-h_{L}\right)}
$$

Dado que $\left(1+\mathrm{h}_{M}\right)$ es positivo, $\mathrm{h}_{L}$, es menor o igual a uno, $\mathrm{y}_{1}$ y $\mathrm{L}_{1}^{d}$ son positivas, es claro que bajo condiciones usuales de estabilidad --la propensión al gasto menor que uno-- la pendiente de la IS es positiva (e infinita $\mathrm{si}_{L^{\prime}}$ es igual a uno). La pendiente de la LM está, por su parte dado por:

$$
\frac{d L}{d Y} / \overline{\bar{L}} M \frac{M_{1}^{d}+h_{M}\left(1-h_{L}\right) L_{1}^{d}}{1 / \propto(1-e)+h_{M}\left(1-h_{L}\right)}
$$

Dada la condición (13) y el hecho que $\mathrm{h}_{A}$ es necesariamente positivo, $\mathrm{h}_{M}$ es siempre mayor que menos uno y es fácil checar que la pendiente de la LM es, en general, positiva.

Por otra parte, si se define como ZZ al conjunto de puntos que corresponden a cierto objetivo de equilibrio externo (o sea un nivel dado de reservas en divisas), la pendiente de esta curva se obtiene a partir de la ecuación (8): 


$$
\frac{d L}{d Y} / \overline{\bar{Z} Z} \frac{L_{1}^{d}+h_{L^{\prime}} L_{1}^{d}-Z_{1}}{h_{L^{\prime}}}
$$

Esta pendiente es positiva o negativa conforme la elasticidad-ingreso de la demanda de préstamos externos sea más alta o más baja que la elasticidad correspondiente del déficit en cuenta corriente. Finalmente, se puede comprobar que la pendiente de la IS es mayor a la pendiente de la demanda nocional de crédito si la propensión al gasto satisface la siguiente condición:

$$
\mathrm{A}_{1}^{d} \leq 1+\mathrm{Z}_{1}
$$

Bajo condiciones usuales de estabilidad, esta condición debe verificarse.

Como se aprecia en la gráfica 1, la intersección de las curvas IS y LM definen un punto de equilibrio interno $\mathrm{E}^{*}$ para la economía. Si este punto se encuentra por debajo de la curva de demanda nocional de crédito, existe desequilibrio por exceso de demanda en el mercado de fondos prestables. Si se encuentra por encima de esta curva, el desequilibrio es por exceso de oferta. Pero en este último caso el punto que define el estado interno de la economía ya no está dado por la intersección de las curvas IS y LM sino por la intersección de las curvas IS y $\mathrm{L}^{d}$ (el punto $\bar{E}$ en la gráfica 1).

La curva ZZ separa al plano en dos regiones: debajo de esta curva (para valores bajos de L), la demanda de créditos internos es alta y existe un superávit en balanza de pagos; por arriba de la curva existe déficit.

\section{III.3 La Política Monetaria en el Corto Plazo.}

Una reducción en el encaje desplaza la LM hacia arriba y no afecta ni a la IS ni a la ZZ. Un aumento en la tasa pasiva desplaza la ZZ hacia arriba. Su efecto sobre la IS es probablemente pequeño dado que la elasticidad interés de la demanda efectiva de bienes es menor a la elasticidad correspondiente de la demanda nocional ${ }^{10}$ : al aumentar $\mathrm{r}_{p}$ se reduce $\mathrm{L}^{d}$ y se reduce el impacto negativo del término de derrame, lo que permite compensar en cierta medida la reducción simultánea en $\mathrm{A}^{d}$. El impacto sobre la LM de una alza en $\mathrm{r}_{p}$ puede ser paradójico: es fácil checar con la ecuación

\footnotetext{
${ }^{10}$ La elasticidad interés de la demanda efectiva de bienes es $\mathrm{A}_{2}^{d}-\left(1+\mathrm{h}_{M}\right)\left(1-\mathrm{h}_{L^{\prime}}\right) \mathrm{L}_{2}^{d}$
} 
(17) que si $\mathrm{D}$ y $\mathrm{M}^{d}$ aumentan más con $\mathrm{r}_{p}$ que lo que puede reducirse el término de derrame -es probable que esto ocurra- la LM se desplaza hacia arriba en la gráfica $1 \mathrm{y}$ el nivel de ingreso aumenta. Incrementar la tasa pasiva de interés tiene entonces un impacto expansionista. Pero para simplificar el análisis gráfico, supondremos ahora que cambios en $\mathrm{r}_{p}$ no afectan ni la IS ni la LM. Este supuesto podría fácilmente eliminarse sin que se modifique sustancialmente el análisis.

\section{Gráfica 1}

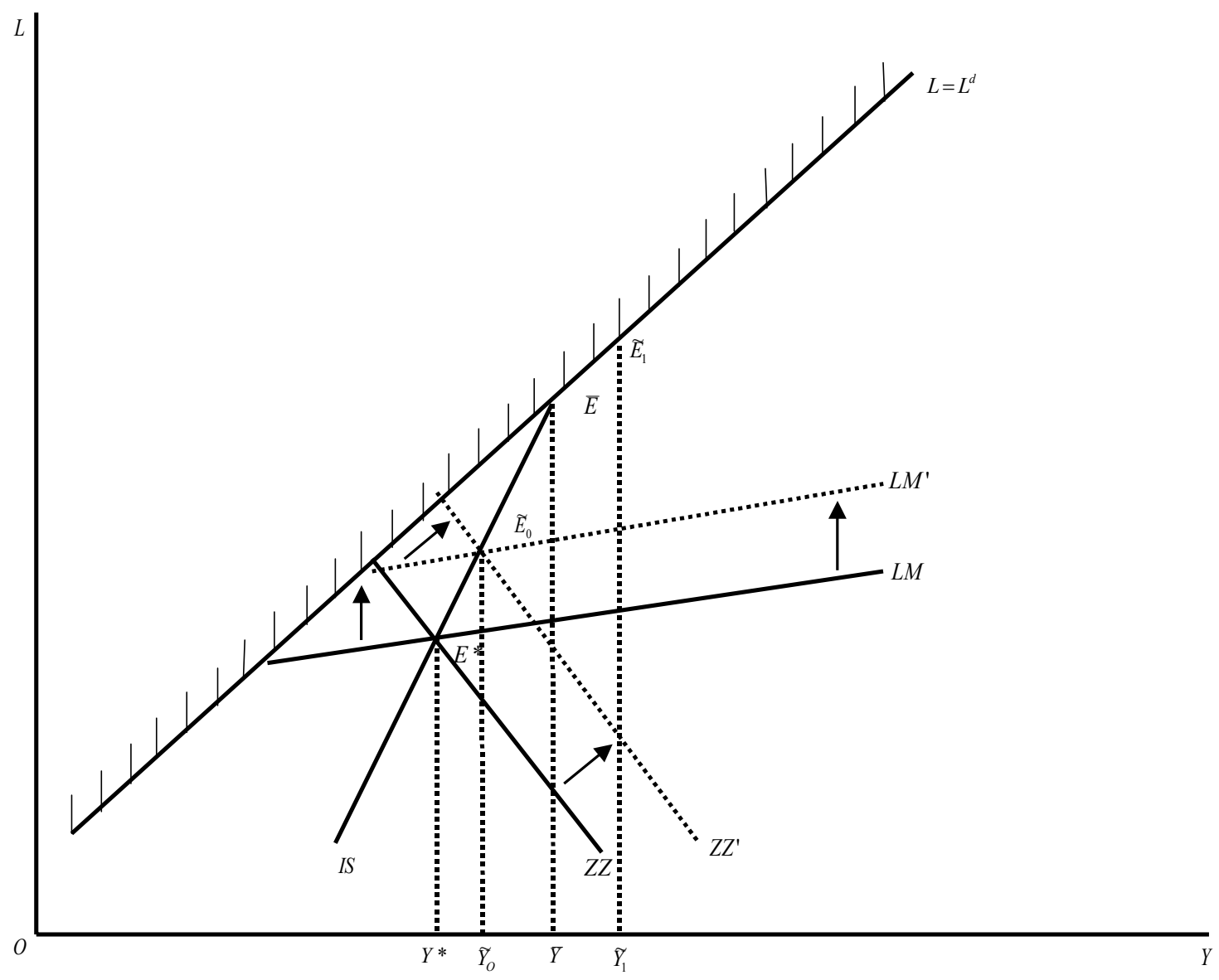

El problema de política a corto plazo se puede entonces sintetizar de la forma siguiente: si el nivel deseado de ingreso, $\tilde{Y}$, se encuentra por abajo del nivel $\bar{Y}$ que corresponde al punto de intersección de las curvas $\mathrm{L}^{d}$ e IS, un ajuste en el encaje permite desplazar la LM hasta alcanzar el nivel deseado de ingreso. Un ajuste en la tasa pasiva permite, por otra parte, desplazar la curva ZZ para conservar el equilibrio externo. En el caso representado en la gráfica 1, si $\mathrm{Y}_{0}$ es el objetivo de ingresos, una reducción en el encaje permite aumentar la oferta de créditos y activar la economía hasta alcanzar 
el nivel deseado $\mathrm{Y}_{0}$. Un aumento en la tasa pasiva es, por otra parte, necesario para que se pueda conservar el equilibrio en la balanza de pagos. La política monetaria es entonces efectiva para permitir alcanzar el punto de equilibrio deseado $\mathrm{E}$.

Sin embargo, la política monetaria no permitirá alcanzar este punto si el ingreso deseado, por ejemplo el nivel $\tilde{Y}_{1}$ en la gráfica 1 , se encuentra por arriba del nivel $\bar{Y}^{11}$. En este caso, una vez alcanzado el nivel $\bar{E}$, reducciones adicionales en el encaje son inefectivas, dado que entonces existe exceso de oferta de fondos. En esta situación la economía se encuentra en una trampa interna de liquidez en la cual cualquier inyección de liquidez es absorbida a través de una mayor demanda de bonos y no tiene efecto alguno sobre el gasto. La única forma de salir del "bache" económico es mediante el uso de la política fiscal, desplazando la IS hacia la derecha.

El segundo caso de inefectividad de la política monetaria se presenta cuando el efecto de derrame sobre la demanda externa de préstamos es completo: $\mathrm{h}_{L^{\prime}}$, igual a uno. Es fácil comprobar a partir de la ecuación (18), que la curva IS es vertical: el nivel de ingreso es inalterable por vía monetaria. Este caso corresponde a una trampa externa de liquidez; cualquier alteración en la oferta interna de liquidez es compensada por variaciones inversas en la oferta externa de fondos.

El último caso de inefectividad ocurre cuando tanto la demanda de préstamos como la demanda de valores mexicanos por extranjeros son inelásticas a la tasa de interés. Queda claro entonces, que cambios en $\mathrm{r}_{p}$ no permitirían alterar la curva $\mathrm{ZZ} \mathrm{y}$, por lo tanto, el objetivo de equilibrio externo sería en general inalcanzable.

Antes de terminar esta discusión, se debe subrayar que la política monetaria puede ser efectiva aún si la elasticidad interés del gasto es nula: la pendiente de la IS puede ser positiva aún si el gasto es insensitivo a variaciones en la tasa de interés sobre préstamos. Esta es una ventaja relativa de una situación de desequilibrio por racionamiento de créditos que es enfatizada por Koelher (1968) y por Bazdrech (1973).

\footnotetext{
${ }^{11}$ Esta situación puede presentarse como el resultado de un desplazamiento de la curva IS hacia la izquierda, causado, por ejemplo, por una caída en las expectativas de inversión.
} 


\section{III.4 El caso de una economía inestable.}

Es interesante examinar cual sería la situación si la condición de estabilidad (21) no se cumpliera. En este caso, la pendiente de la IS es menor a la pendiente de la $\mathrm{L}^{d}$, como se representa en la gráfica 2.

\section{Gráfica 2}



Cualquier objetivo de ingreso, Y, mayor al nivel $\bar{Y}$ es entonces alcanzable por la vía monetaria: al reducir en encaje aumenta la oferta de fondos pero se incrementa aún más su demanda, una vez que ha aumentado el nivel de ingreso; se puede entonces conservar una situación de exceso de demanda en el mercado de fondos prestables que permite absorber toda la inyección de liquidez, canalizándose ésta principalmente hacia el gasto. 
Es interesante destacar que al caer la economía en el punto $\bar{E}$, en donde la IS intersecta la $\mathrm{L}^{d}$, la restricción en el mercado de fondos se hace por el lado de la demanda y una inyección de liquidez no tiene efecto alguno sobre el gasto, ya que se queda congelada dentro del sistema bancario. Una pequeña alteración en el ingreso incrementaría la demanda de fondos suficientemente para que la restricción provenga por el lado de la oferta, permitiendo así el "despegue" de la economía. Pero en la ausencia de este impacto, la economía está condenada a permanecer en $\bar{E}$. Este último punto tiene una propiedad interesante: es posible aproximarse a él tanto como se pueda y alejarse mediante medidas monetarias; pero al caer en él, ya no hay escape posible por la vía puramente monetaria.

\section{III.5 Una extensión con capital de trabajo}

Se puede extender fácilmente el análisis al caso en que la capacidad productiva, a través del capital de trabajo, es afectada por las condiciones crediticias. Si y es la cantidad producida:

$$
\mathrm{y}=\min \left\{\mathrm{y}^{d}, \mathrm{y}^{0}\right\}
$$

en donde $\mathrm{y}^{d}$, el producto demandado corresponde a la curva IS y se obtiene como antes a partir de la ecuación (3) y $\mathrm{y}^{0}$, el producto ofrecido, es la suma de una oferta nocional de "pleno empleo", $\mathrm{y} f$, y de un efecto de derrame del mercado crediticio:

$$
\mathrm{y}^{0}=\mathrm{y}^{f}-\mathrm{h}_{y}\left(\mathrm{~L}^{d}\left(\mathrm{y}, \mathrm{r}_{p}\right)-\mathrm{L}\right)
$$

Esta curva de oferta intersecta la $\mathrm{L}^{d}$ en el punto de pleno empleo, $\mathrm{y}^{f}$; para $\mathrm{L}$ menor a $\mathrm{L}^{d}, \mathrm{y}^{0}$ es menor a $\mathrm{y}^{f}$; y para $\mathrm{L}$ mayor a $\mathrm{L}^{d}, \mathrm{y}^{0}$ sigue igual a $\mathrm{y}^{f}$. Dos casos pueden presentarse según la pendiente de la $\mathrm{y}^{0}$ (para valores de $\mathrm{L}$ menores a $\mathrm{L}^{d}$ ) sea menor o mayor a la pendiente de la IS. Consideremos primero el caso en qué cambios en el crédito tienen un impacto muy limitado en la oferta de bienes y por lo tanto la pendiente de la $\mathrm{y}^{0}$ es mayor a la de la IS. Este caso aparece en la gráfica 3. Si la IS intersecta la $\mathrm{L}^{d}$ a un nivel de ingreso $\bar{y}_{0}$ menor a $\mathrm{y}^{f}$, toda la curva $\mathrm{y}^{0}$ esta a la derecha de la IS, y, conforme la condición (22), el equilibrio se obtiene, como antes, en el punto de intersección de la IS y de la LM. Si la IS intersecta la $\mathrm{L}^{d}$ a un nivel de ingreso $\bar{y}$, mayor a $\mathrm{y}^{f}$, el ingreso real se obtiene entonces, para valores elevados del crédito, en la intersección de la y y de la LM; y para valores del crédito por debajo de $\bar{L}$, que corresponde a la intersección de la IS y de la y ${ }^{0}$, el 
equilibrio esta ubicado sobre la IS. Pero en uno y otro caso es cierto que una restricción crediticia (un desplazamiento de la LM hacia abajo) hace disminuir la demanda de bienes mas que la oferta y contribuye por lo tanto a reducir eventuales presiones inflacionarias.

\section{Gráfica 3}

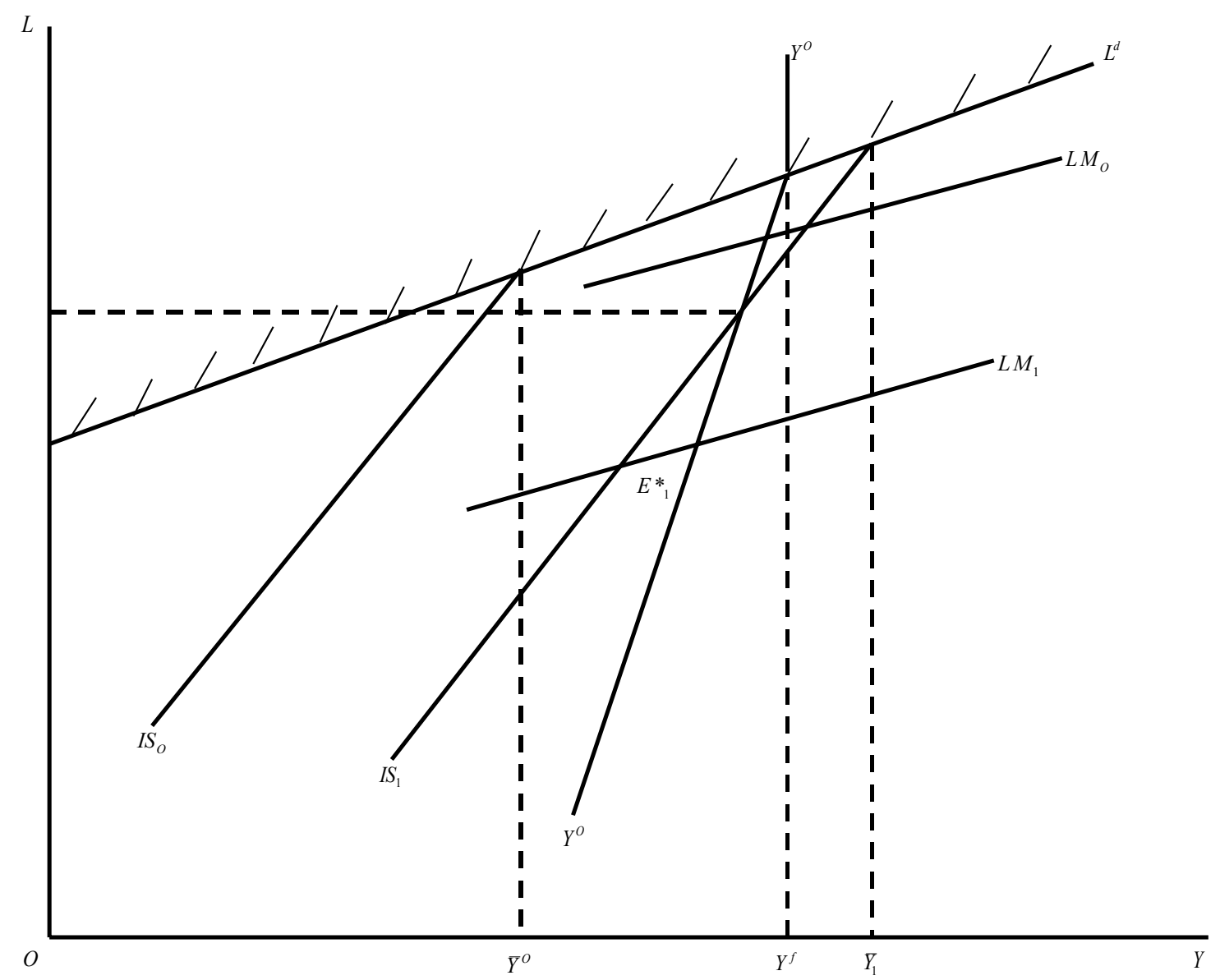

Veamos ahora que pasaría sí cambios en el crédito tienen un fuerte impacto sobre la oferta de bienes, de tal forma que la pendiente de la $\mathrm{y}^{0}$ es menor a la de la IS, tal como aparece en la gráfica 4. El caso más interesante de analizar en detalle ocurre cuando la IS intersecta la $\mathrm{L}^{d}$ en $\bar{y}_{0}$ mayor a $\mathrm{y}^{f}$, de tal forma que la demanda de bienes supera a la oferta y existe una brecha inflacionaria, Si la LM intersecta la IS en el nivel máximo de ingreso, $\bar{y}$, la brecha inflacionaria entre demanda y oferta es $1_{0}$. Si el gobierno reduce el crédito, con el fin de disminuir las presiones inflacionarias, se observa en la gráfica 4 que al desplazarse hacia abajo la LM, primero disminuye el tamaño de la brecha, pasa por un mínimo cuando el nivel de crédito coincide con la intersección de la $\mathrm{y}^{0} \mathrm{y}$ de la $\mathrm{L}^{d}$, y luego vuelve a 
aumentar. En este caso, la brecha inflacionaria sigue existiendo y la política monetaria por si sola es inefectiva para restablecer el equilibrio interno; más aún, una política contraccionista demasiado severa puede ser contraproducente, dado que puede empeorar en vez de mejorar el alza de los precios.

\section{Gráfica 4}



\section{Conclusión}

El modelo que se presentó aquí permitió identificar cuales son los límites y los alcances de la política monetaria en un sistema financiero en desequilibrio como el de México. Se vio en particular qué estímulos monetarios podían ser inefectivos para reactivar la economía sí las expectativas eran muy pesimistas; y esto aún si el gasto privado era elástico con respecto a la tasa de interés. Por otra parte, si las expectativas no eran demasiado pesimistas, medidas monetarias podían ser efectivas aún sí el gasto privado era inelástico con respecto a la tasa de interés. Se vio también qué políticas monetarias restrictivas destinadas a frenar presiones inflacionarias debidas a un exceso de demanda podían 
provocar resultados exactamente opuestos a los deseados, ya que podían afectar la oferta de bienes más que la demanda. Todos estos resultados son propios de un modelo en desequilibrio.

Este modelo puede ser extendido en varias direcciones: una primera extensión constituye el incluir la tasa de inflación como variable determinante en las funciones de demanda de bienes y de activos monetarios y financieros, haciendo depender al ingreso de la tasa de inflación a través de una relación similar a la curva de Phillips. Otra extensión es considerar el efecto que puede causar el riesgo cambiario. Cambios en las expectativas de devaluación alteran grandemente las demandas de activos financieros y con ello el estado de liquidez de la economía y el gasto. Si estas expectativas dependen de los cambios habidos en las reservas de divisas, es claro que el proceso puede fácilmente volverse inestable. Una última extensión consiste en pasar del corto al mediano plazo, incluyendo entonces variables fiscales como instrumentos de política y endogeneizando la determinación de la tasa activa de interés a través de un proceso de ajuste lento al equilibrio en el mercado de fondos prestables.

La estimación econométrica del modelo presenta algunos problemas importantes. En primer lugar, al estimar los efectos de derrame, es necesario realizar regresiones entre el crédito y otras variables como el gasto y las demandas de activos monetarios y financieros; pero el grado de colinealidad entre estas variables puede ser muy alto, lo que puede disminuir la precisión de las estimaciones. Por otra parte, es necesario, para poder estimar la demanda nocional de crédito interno, aíslas aquellos periodos en los cuales existe equilibrio en el mercado de fondos prestables o desequilibrio por exceso de oferta. Se requiere, por lo tanto, conocer aquellas épocas. Es también necesario que estas últimas sean lo suficientemente frecuentes y prolongadas para permitir derivar en forma estadísticamente confiable una función de demanda de crédito. Sin embargo, es indispensable superar estas dificultades si se quiere obtener un modelo econométrico realmente apropiado para la toma de decisiones en materia de política monetaria. 


\section{B I B L I O G R A F I A}

BAZDRECH, Carlos. (1973) “La Política Monetaria Mexicana; una primera aproximación”, en Solís, L. “La Economía Mexicana IV, México, D.F., Fondo de Cultura Económica.

BARRO, R.J. y H.I. GROSSMAN (1971) “A General Disequilibrium Model of Income and Employment", American Economic Review. March 1971.

CLOWER, R.W. (1965), “The Keynesian Conterrevolution: A Theoretical Appraisal” en F.H. Hanh y F.P.R Brechling (eds.). The Theory of interest Rates, London: McMillian.

IZE, Alain (1979) “La Política Monetaria en México: una Reseña” en Banco de México, Subdirección de Investigación Económica y Bancaria. Documento de Investigación No. 5.

Koehler, John E. (1968) "Economic Policy making with limited information: the process of macroeconomic control in Mexico". The Rand Corporation.

KORLIRAS, Panayotis, G. (1975) “A Disequilibrium Macroeconomic Model” Quarterly Journal of Economics, feb. 75 , p. 56.

LEIJONHUFVUD, A. (1968), On Keynesian Economics and the Economics of Keynes, New York: Oxford University Press.

MALINVAUD, E. (1977) The Theory of Unemployment Reconsideres New York: John Wiley.

MCKINNON, Ronald I. Money and capital in Economic Development. The Brooking Institution, 1973. 
BANCO DE MEXICO, S.A.

SUBDIRECCION DE INVESTIGACION ECONOMICA

SERIE DOCUMENTOS DE INVESTIGACION

1. ESTRUCTURA FINANCIERA Y EXPERIENCIA CAMBIARIA: MEXICO 1954-1977.

Guillermo Ortiz

Octubre, 1978.

2. EL FINANCIAMIENTO DEL GASTO PUBLICO EN UNA ECONOMIA EN CRECIMIENTO: EL CASO DE MEXICO.

Alain Ize.

Noviembre, 1978.

3. ALGUNOS ASPECTOS DEL ENDEUDAMIENTO PUBLICO EXTERNO EN MEXICO.

Ernesto Zedillo.

Diciembre, 1978.

4. UNA APLICACIÓN DEL MODELO BAYESIANO DE DECISION EN EL ANALISIS DE FUNCIONES DE PRODUCCION AGRICOLA.

Héctor E. González Méndez.

Diciembre, 1978.

5. POLITICA MACROECONOMICA EN EL CORTO PLAZO: UNA RESEÑA.

Alain Ize.

Marzo, 1979.

6. ESTUDIOS DE MONEDA Y BANCA Y POLITICA MONETARIA SOBRE MEXICO: SELECCIÓN BIBLIOGRAFICA DE 1943 A 1978.

Abril, 1979.

7. COMERCIO EXTERIOR MEXICO-ESTADOS UNIDOS: PROBLEMAS DE COMPARABILIDAD ESTADISTICA.

Jorge Carriles Rubio.

Mayo, 1979.

8. EXPLOTACION OPTIMA DE RESERVAS PETROLERAS EN UN CONTEXTO MACROECONOMICO.

José Córdoba.

Mayo, 1979. 
9. ASPECTOS DEFLACIONARIOS DE LA DEVALUACION DEL PESO MEXICANO DE 1976.

José Córdoba y Guillermo Ortiz

Mayo, 1979.

10. EXTRACCION OPTIMA DE PETROLEO Y ENDEUDAMIENTO EXTERNO: EL CASO DE MEXICO.

Ernesto Zedillo.

Junio, 1979.

11. IMPUESTOS DIRECTOS: PROGRESIVIDAD OPTIMA.

Jesús Seade.

Septiembre, 1979.

12. OPCIONES DE POLITICA ECONOMICA 1979-1982.

Sócrates Rizo y Leopoldo Solís.

Septiembre, 1978.

13. INTERMEDIARIOS FINANCIEROS Y MERCADOS IMPERFECTOS DE CAPITAL.

Guillermo Ortiz.

Septiembre, 1979.

14. ESTIMACIONES DE EQUILIBRIO GENERAL DE LOS EFECTOS DE LAS DISTORSIONES EN LOS MERCADOS DE FACTORES: EL CASO DE MEXICO.

José Sidaoui y Richard H. Sines.

Octubre, 1979.

15. UN ANALISIS DE LA INFLACION EN MEXICO.

Alain Ize.

Octubre, 1979.

16. ANALISIS DE LOS COMPONENTES DEL CAMBIO ESTRUCTURAL CON UN MODELO DE EQUILIBRIO GENERAL, 1970-75.

José Sidaoui y Richard H. Sines.

Enero, 1980.

17. TIPOS DE CAMBIO FLOTANTES Y DESLIZ CAMBIARIO: LAS EXPERIENCIAS DE ALGUNOS PAISES EN DESARROLLO.

Guillermo Ortiz y Leopoldo Solís.

Enero, 1980. 
18. UN MODELO DE INFLACION Y CRECIMIENTO EN UNA ECONOMIA CAPITALISTA EN DESARROLLO.

Alain Ize.

Enero, 1980.

19. CRECIMIENTO E INFLACION: ALTERNATIVAS CAMBIARIAS PARA MEXICO.

Guillermo Ortiz y Leopoldo Solís.

Febrero, 1980.

20. COMPORTAMIENTO DE LA CAPTACION BANCARIA EN MEXICO.

Héctor E. González Méndez.

Mayo, 1980.

21. LA ENCUESTA DE TURISMO RECEPTIVO, REPORTE METODOLOGICO.

Alberto Vargas Aguayo.

Junio, 1980 .

22. AJUSTE ESTACIONAL DE UNA SERIE DE TIEMPO MEDIANTE EL USO COMPLEMENTARIO DE METODOS TRADICIONALES Y LA TECNICA DE BOX-JENKINS.

Gabriel Vera Ferrer y Víctor M. Guerrero.

Junio, 1980 .

23. DISTRIBUCION DEL FINANCIAMIENTO OTORGADO POR EL SISTEMA BANCARIO MEXICANO A LA BANCA PRIVADA Y MIXTA.

Víctor M. Guerrero y Gabriel Vera Ferrer.

Julio, 1980.

24. LA MIGRACION INDOCUMENTADA A ESTADOS UNIDOS: UN NUEVO ENFOQUE. Juan Díez-Canedo.

Julio, 1980.

25. UN MODELO FINANCIERO DE DESEQUILIBRIO A CORTO PLAZO PARA LA ECONOMIA MEXICANA.

Alain Ize.

Julio, 1980. 